\title{
On the spectral norm of $r$-circulant matrices with the Pell and Pell-Lucas numbers
}

\author{
Ramazan Türkmen ${ }^{1 *}$ and Hasan Gökbaş²
}

${ }^{\text {*Correspondence: }}$

rturkmen@selcuk.edu.tr

${ }^{1}$ Science Faculty, Selcuk University, Konya, 42031, Turkey

Full list of author information is

available at the end of the article

\begin{abstract}
Let us define $A=C_{r}\left(a_{0}, a_{1}, \ldots, a_{n-1}\right)$ to be a $n \times n r$-circulant matrix. The entries in the first row of $A=C_{r}\left(a_{0}, a_{1}, \ldots, a_{n-1}\right)$ are $a_{i}=P_{i}, a_{i}=Q_{i}, a_{i}=P_{i}^{2}$ or $a_{i}=Q_{i}^{2}$

$(i=0,1,2, \ldots, n-1)$, where $P_{i}$ and $Q_{i}$ are the ith Pell and Pell-Lucas numbers, respectively. We find some bounds estimation of the spectral norm for $r$-Circulant matrices with Pell and Pell-Lucas numbers.
\end{abstract}

Keywords: Pell numbers; Pell-Lucas numbers; r-circulant matrix; spectral norm

\section{Introduction}

Special matrices is a widely studied subject in matrix analysis. Especially special matrices whose entries are well-known number sequences have become a very interesting research subject in recent years and many authors have obtained some good results in this area. For example, Bahşi and Solak have studied the norms of $r$-circulant matrices with the hyperFibonacci and Lucas numbers [1], Bozkurt and Tam have obtained some results belong to determinants and inverses of $r$-circulant matrices associated with a number sequence [2], Shen and Cen have made a similar study by using $r$-circulant matrices with the Fibonacci and Lucas numbers $[3,4]$ and He et al. have established on the spectral norm inequalities on $r$-circulant matrices with Fibonacci and Lucas numbers [5].

Lots of article have been written so far, which concern estimates for spectral norms of circulant and $r$-circulant matrices, which have connections with signal and image processing, time series analysis and many other problems.

In this paper, we derive expressions of spectral norms for $r$-circulant matrices. We explain some preliminaries and well-known results. We thicken the identities of estimations for spectral norms of $r$-circulant matrices with the Pell and Pell-Lucas numbers.

The Pell and Pell-Lucas sequences $P_{n}$ and $Q_{n}$ are defined by the recurrence relations

$$
P_{0}=0, \quad P_{1}=1, \quad P_{n}=2 P_{n-1}+P_{n-2} \quad \text { for } n \geq 2
$$

and

$$
Q_{0}=2, \quad Q_{1}=2, \quad Q_{n}=2 Q_{n-1}+Q_{n-2} \quad \text { for } n \geq 2 .
$$

(c) 2016 Türkmen and Gökbaş. This article is distributed under the terms of the Creative Commons Attribution 4.0 International License (http://creativecommons.org/licenses/by/4.0/), which permits unrestricted use, distribution, and reproduction in any medium, provided you give appropriate credit to the original author(s) and the source, provide a link to the Creative Commons license, and indicate if changes were made. 
If we start from $n=0$, then the Pell and Pell-Lucas sequence are given by

$\begin{array}{llllllllll}n: & 0 & 1 & 2 & 3 & 4 & 5 & 6 & 7 & \ldots \\ P_{n}: & 0 & 1 & 2 & 5 & 12 & 29 & 70 & 169 & \ldots \\ Q_{n}: & 2 & 2 & 6 & 14 & 34 & 82 & 198 & 478 & \ldots\end{array}$

The following sum formulas for the Pell and Pell-Lucas numbers are well known [6, 7]:

$$
\sum_{k=1}^{n} P_{k}^{2}=\frac{P_{n} P_{n+1}}{2}
$$

and

$$
\sum_{k=1}^{n} Q_{k}^{2}=\frac{Q_{2 n+1}+2(-1)^{n}-4}{2}
$$

A matrix $C=\left[c_{i j}\right] \in M_{n, n}(\mathbb{C})$ is called a $r$-circulant matrix if it is of the form

$$
c_{i j}= \begin{cases}c_{j-i}, & j \geq i, \\ r c_{n+j-i}, & j<i .\end{cases}
$$

Obviously, the $r$-circulant matrix $C$ is determined by the parameter $r$ and its first row elements $c_{0}, c_{1}, \ldots, c_{n-1}$, thus we denote $C=C_{r}\left(c_{0}, c_{1}, \ldots, c_{n-1}\right)$. Especially, let $r=1$, the matrix $C$ is called a circulant matrix [3].

The Euclidean norm of the matrix $A$ is defined as

$$
\|A\|_{E}=\left(\sum_{i, j=1}^{n}\left|a_{i j}\right|^{2}\right)^{1 / 2} .
$$

The singular values of the matrix $A$ are

$$
\sigma_{i}=\sqrt{\lambda_{i}\left(A^{*} A\right)}
$$

where $\lambda_{i}$ is an eigenvalue of $A^{*} A$ and $A^{*}$ is conjugate transpose of matrix $A$. The square roots of the maximum eigenvalues of $A^{*} A$ are called the spectral norm of $A$ and are induced by $\|A\|_{2}$.

The following inequality holds:

$$
\frac{1}{\sqrt{n}}\|A\|_{E} \leq\|A\|_{2} \leq\|A\|_{E}
$$

Define the maximum column length norm $c_{1}$, and the maximum row length norm $r_{1}$ of any matrix $A$ by

$$
r_{1}(A)=\max _{i} \sqrt{\sum_{j}\left|a_{i j}\right|^{2}}
$$


and

$$
c_{1}(A)=\max _{j} \sqrt{\sum_{i}\left|a_{i j}\right|^{2}}
$$

respectively. Let $A, B$, and $C$ be $m \times n$ matrices. If $A=B \circ C$ then

$$
\|A\|_{2} \leq r_{1}(B) c_{1}(C) \quad[8]
$$

\section{Result and discussion}

Theorem 1 Let $A=C_{r}\left(P_{0}, P_{1}, \ldots, P_{n-1}\right)$ be a $r$-circulant matrix, where $r \in \mathbb{C}$. We have

(i) $|r| \geq 1, \quad \sqrt{\frac{P_{n} P_{n-1}}{2}} \leq\|A\|_{2} \leq|r| \sqrt{(n-1) \frac{P_{n} P_{n-1}}{2}}$,

(ii) $|r|<1, \quad|r| \sqrt{\frac{P_{n} P_{n-1}}{2}} \leq\|A\|_{2} \leq \sqrt{(n-1) \frac{P_{n} P_{n-1}}{2}}$.

Proof The matrix $A$ is of the form

$$
A=\left[\begin{array}{ccccc}
P_{0} & P_{1} & \ldots & P_{n-2} & P_{n-1} \\
r P_{n-1} & P_{0} & \ldots & P_{n-3} & P_{n-2} \\
\vdots & \vdots & \ddots & \vdots & \vdots \\
r P_{2} & r P_{3} & \ldots & P_{0} & P_{1} \\
r P_{1} & r P_{2} & \ldots & r P_{n-1} & P_{0}
\end{array}\right]
$$

Then we have

$$
\|A\|_{E}^{2}=\sum_{i=0}^{n-1}(n-i) P_{i}^{2}+\sum_{i=1}^{n-1} i|r|^{2} P_{i}^{2}
$$

hence, when $|r| \geq 1$ we obtain

$$
\|A\|_{E}^{2} \geq \sum_{i=0}^{n-1}(n-i) P_{i}^{2}+\sum_{i=1}^{n-1} i P_{i}^{2}=n \sum_{i=0}^{n-1} P_{i}^{2}=n \frac{P_{n} P_{n-1}}{2}
$$

that is,

$$
\frac{1}{\sqrt{n}}\|A\|_{E} \geq \sqrt{\frac{P_{n} P_{n-1}}{2}} \Rightarrow\|A\|_{2} \geq \sqrt{\frac{P_{n} P_{n-1}}{2}} .
$$

On the other hand, let the matrices $B$ and $C$ be

$$
B=\left[\begin{array}{ccccc}
P_{0} & 1 & \ldots & 1 & 1 \\
r & P_{0} & \ldots & 1 & 1 \\
\vdots & \vdots & \ddots & \vdots & \vdots \\
r & r & \ldots & P_{0} & 1 \\
r & r & \ldots & r & P_{0}
\end{array}\right] \text { and } C=\left[\begin{array}{ccccc}
P_{0} & P_{1} & \ldots & P_{n-2} & P_{n-1} \\
P_{n-1} & P_{0} & \ldots & P_{n-3} & P_{n-2} \\
\vdots & \vdots & \ddots & \vdots & \vdots \\
P_{2} & P_{3} & \ldots & P_{0} & P_{1} \\
P_{1} & P_{2} & \ldots & P_{n-1} & P_{0}
\end{array}\right]
$$


such that $A=B \circ C$. Then

$$
\begin{aligned}
& r_{1}(B)=\max _{i} \sqrt{\sum_{j}\left|b_{n j}\right|^{2}}=\sqrt{|r|^{2}(n-1)}=|r| \sqrt{(n-1)} \text { and } \\
& c_{1}(C)=\max _{j} \sqrt{\sum_{i}\left|c_{i n}\right|^{2}}=\sqrt{\sum_{i=0}^{n-1} P_{i}^{2}}=\sqrt{\frac{P_{n} P_{n-1}}{2}} .
\end{aligned}
$$

We have

$$
\|A\|_{2} \leq|r| \sqrt{(n-1) \frac{P_{n} P_{n-1}}{2}} .
$$

When $|r|<1$ we also obtain

$$
\|A\|_{E}^{2} \geq \sum_{i=0}^{n-1}(n-i)|r|^{2} P_{i}^{2}+\sum_{i=1}^{n-1} i|r|^{2} P_{i}^{2}=n|r|^{2} \frac{P_{n} P_{n-1}}{2},
$$

that is,

$$
\frac{1}{\sqrt{n}}\|A\|_{E} \geq|r| \sqrt{\frac{P_{n} P_{n-1}}{2}} \Rightarrow\|A\|_{2} \geq|r| \sqrt{\frac{P_{n} P_{n-1}}{2}} .
$$

On the other hand, let the matrices $B$ and $C$ be

$$
B=\left[\begin{array}{ccccc}
P_{0} & 1 & \ldots & 1 & 1 \\
r & P_{0} & \ldots & 1 & 1 \\
\vdots & \vdots & \ddots & \vdots & \vdots \\
r & r & \ldots & P_{0} & 1 \\
r & r & \ldots & r & P_{0}
\end{array}\right] \text { and } C=\left[\begin{array}{ccccc}
P_{0} & P_{1} & \ldots & P_{n-2} & P_{n-1} \\
P_{n-1} & P_{0} & \ldots & P_{n-3} & P_{n-2} \\
\vdots & \vdots & \ddots & \vdots & \vdots \\
P_{2} & P_{3} & \ldots & P_{0} & P_{1} \\
P_{1} & P_{2} & \ldots & P_{n-1} & P_{0}
\end{array}\right]
$$

such that $A=B \circ C$. Then

$$
\begin{aligned}
& r_{1}(B)=\max _{i} \sqrt{\sum_{j}\left|b_{i j}\right|^{2}}=\sqrt{\sum_{j=0}^{n-1}\left|b_{n j}\right|^{2}}=\sqrt{n-1} \text { and } \\
& c_{1}(C)=\max _{j} \sqrt{\sum_{i}\left|c_{i j}\right|^{2}}=\sqrt{\sum_{i=0}^{n-1}\left|c_{i n}\right|^{2}}=\sqrt{\sum_{i=0}^{n-1} P_{i}^{2}}=\sqrt{\frac{P_{n} P_{n-1}}{2}} .
\end{aligned}
$$

We have

$$
\|A\|_{2} \leq \sqrt{(n-1) \frac{P_{n} P_{n-1}}{2}} .
$$

Thus, the proof is completed.

Corollary 2 Let $A=C_{r}\left(P_{0}^{2}, P_{1}^{2}, \ldots, P_{n-1}^{2}\right)$ be a $r$-circulant matrix, where $r \in \mathbb{C},|r| \geq 1$; we have 


$$
\|A\|_{2} \leq(n-1)|r| \frac{P_{n} P_{n-1}}{2},
$$

where $\|\cdot\|_{2}$ is the spectral norm and $P_{n}$ denotes the nth Pell number.

Proof Since $A=C_{r}\left(P_{0}^{2}, P_{1}^{2}, \ldots, P_{n-1}^{2}\right)$ is a $r$-circulant matrix, if the matrices $B=C_{r}\left(P_{0}, P_{1}\right.$, $\left.\ldots, P_{n-1}\right)$ and $C=C\left(P_{0}^{2}, P_{1}^{2}, \ldots, P_{n-1}^{2}\right)$ we get $A=B \circ C$; thus, we obtain

$$
\|A\|_{2} \leq(n-1)|r| \frac{P_{n} P_{n-1}}{2} .
$$

Theorem 3 Let $A=C_{r}\left(Q_{0}, Q_{1}, \ldots, Q_{n-1}\right)$ be a $r$-circulant matrix, where $r \in \mathbb{C}$.

$$
\begin{aligned}
& \text { (i) }|r| \geq 1, \quad \begin{cases}\sqrt{\frac{Q_{2 n-1}+6}{2}} \leq\|A\|_{2} \leq|r| \sqrt{n \frac{Q_{2 n-1}+6}{2}}, & \text { nodd, } \\
\sqrt{\frac{Q_{2 n-1}+2}{2}} \leq\|A\|_{2} \leq|r| \sqrt{n \frac{Q_{2 n-1}+2}{2}}, & \text { n even, }\end{cases} \\
& \text { (ii) } \quad|r|<1, \quad \begin{cases}|r| \sqrt{\frac{Q_{2 n-1}+6}{2}} \leq\|A\|_{2} \leq \sqrt{n \frac{Q_{2 n-1}+6}{2}}, & \text { nodd, } \\
|r| \sqrt{\frac{Q_{2 n-1}+2}{2}} \leq\|A\|_{2} \leq \sqrt{n \frac{Q_{2 n-1}+2}{2}}, & \text { neven. }\end{cases}
\end{aligned}
$$

Proof The matrix $A$ is of the form

$$
A=\left[\begin{array}{ccccc}
Q_{0} & Q_{1} & \ldots & Q_{n-2} & Q_{n-1} \\
r Q_{n-1} & Q_{0} & \ldots & Q_{n-3} & Q_{n-2} \\
\vdots & \vdots & \ddots & \vdots & \vdots \\
r Q_{2} & r Q_{3} & \ldots & Q_{0} & Q_{1} \\
r Q_{1} & r Q_{2} & \ldots & r Q_{n-1} & Q_{0}
\end{array}\right]
$$

Then we have

$$
\|A\|_{E}^{2}=\sum_{i=0}^{n-1}(n-i) Q_{i}^{2}+\sum_{i=1}^{n-1} i|r|^{2} Q_{i}^{2}
$$

hence, when $|r| \geq 1$ we obtain

$$
\|A\|_{E}^{2} \geq \sum_{i=0}^{n-1}(n-i) Q_{i}^{2}+\sum_{i=1}^{n-1} i Q_{i}^{2}=n \sum_{i=0}^{n-1} Q_{i}^{2}= \begin{cases}\sqrt{n \frac{Q_{2 n-1}+6}{2}}, & n \text { odd } \\ \sqrt{\frac{n Q_{2 n-1}+2}{2},}, & n \text { even }\end{cases}
$$

that is,

$$
\frac{1}{\sqrt{n}}\|A\|_{E} \geq\|A\|_{2} \geq \begin{cases}\frac{\sqrt{\frac{Q_{2 n-1}+6}{2}},}{\sqrt{\frac{Q_{2 n-1}+2}{2}},} & n \text { odd } \\ \sqrt{2} \text { even }\end{cases}
$$

On the other hand, let the matrices $B$ and $C$ be

$$
B=\left[\begin{array}{ccccc}
1 & 1 & \ldots & 1 & 1 \\
r & 1 & \ldots & 1 & 1 \\
\vdots & \vdots & \ddots & \vdots & \vdots \\
r & r & \ldots & 1 & 1 \\
r & r & \ldots & r & 1
\end{array}\right] \text { and } C=\left[\begin{array}{ccccc}
Q_{0} & Q_{1} & \ldots & Q_{n-2} & Q_{n-1} \\
Q_{n-1} & Q_{0} & \ldots & Q_{n-3} & Q_{n-2} \\
\vdots & \vdots & \ddots & \vdots & \vdots \\
Q_{2} & Q_{3} & \ldots & Q_{0} & Q_{1} \\
Q_{1} & Q_{2} & \ldots & Q_{n-1} & Q_{0}
\end{array}\right]
$$


such that $A=B \circ C$. Then

$$
\begin{aligned}
& r_{1}(B)=\max _{i} \sqrt{\sum_{j}\left|b_{i j}\right|^{2}}=\sqrt{\sum_{j=0}^{n-1}\left|b_{n j}\right|^{2}}=\sqrt{|r|^{2}(n-1)+1} \text { and } \\
& c_{1}(C)=\max _{j} \sqrt{\sum_{i}\left|c_{i j}\right|^{2}}=\sqrt{\sum_{i=0}^{n-1}\left|c_{i n}\right|^{2}}=\sqrt{\sum_{i=0}^{n-1} Q_{i}^{2}}= \begin{cases}\sqrt{\frac{Q_{2 n-1}+6}{2}}, & n \text { odd } \\
\sqrt{\frac{Q_{2 n-1}+2}{2}}, & n \text { even. }\end{cases}
\end{aligned}
$$

We have

$$
\|A\|_{2} \leq \begin{cases}\sqrt{\left(|r|^{2}(n-1)+1\right)\left(\frac{Q_{2 n-1}+6}{2}\right)}, & n \text { odd } \\ \sqrt{\left(|r|^{2}(n-1)+1\right)\left(\frac{Q_{2 n-1}+2}{2}\right)}, & n \text { even }\end{cases}
$$

When $|r|<1$ we also obtain

$$
\|A\|_{E}^{2} \geq \sum_{i=0}^{n-1}(n-i)|r|^{2} Q_{i}^{2}+\sum_{i=1}^{n-1} i|r|^{2} Q_{i}^{2}= \begin{cases}|r| \sqrt{n\left(\frac{Q_{2 n-1}+6}{2}\right)}, & n \text { odd } \\ |r| \sqrt{n\left(\frac{Q_{2 n-1}+2}{2}\right),} & n \text { even }\end{cases}
$$

that is,

$$
\frac{1}{\sqrt{n}}\|A\|_{E} \geq\|A\|_{2} \geq \begin{cases}|r| \sqrt{\frac{Q_{2 n-1}+6}{2}}, & n \text { odd } \\ |r| \sqrt{\frac{Q_{2 n-1}+2}{2}}, & n \text { even. }\end{cases}
$$

On the other hand, let the matrices $B$ and $C$ be

$$
B=\left[\begin{array}{ccccc}
1 & 1 & \ldots & 1 & 1 \\
r & 1 & \ldots & 1 & 1 \\
\vdots & \vdots & \ddots & \vdots & \vdots \\
r & r & \ldots & 1 & 1 \\
r & r & \ldots & r & 1
\end{array}\right] \text { and } C=\left[\begin{array}{ccccc}
Q_{0} & Q_{1} & \ldots & Q_{n-2} & Q_{n-1} \\
Q_{n-1} & Q_{0} & \ldots & Q_{n-3} & Q_{n-2} \\
\vdots & \vdots & \ddots & \vdots & \vdots \\
Q_{2} & Q_{3} & \ldots & Q_{0} & Q_{1} \\
Q_{1} & Q_{2} & \ldots & Q_{n-1} & Q_{0}
\end{array}\right]
$$

such that $A=B \circ C$. Then

$$
\begin{aligned}
& r_{1}(B)=\max _{i} \sqrt{\sum_{j}\left|b_{i j}\right|^{2}}=\sqrt{\sum_{j=0}^{n-1}\left|b_{n j}\right|^{2}}=\sqrt{n} \text { and } \\
& c_{1}(C)=\max _{j} \sqrt{\sum_{i}\left|c_{i j}\right|^{2}}=\sqrt{\sum_{i=0}^{n-1}\left|c_{i n}\right|^{2}}=\sqrt{\sum_{i=0}^{n-1} Q_{i}^{2}}= \begin{cases}\frac{\sqrt{\frac{Q_{2 n-1}+6}{2}},}{\sqrt{\frac{Q_{2 n-1+2}}{2}},} & n \text { odd } \\
\sqrt{1} \text { even. }\end{cases}
\end{aligned}
$$

We have

$$
\|A\|_{2} \leq \begin{cases}\sqrt{n \frac{Q_{2 n-1}+6}{2}}, & n \text { odd } \\ \sqrt{n \frac{Q_{2 n-1}+2}{2}}, & n \text { even }\end{cases}
$$

Thus, the proof is completed. 
Corollary 4 Let $A=C_{r}\left(Q_{0}^{2}, Q_{1}^{2}, \ldots, Q_{n-1}^{2}\right)$ be a $r$-circulant matrix, where $r \in \mathbb{C},|r| \geq 1$,

$$
\|A\|_{2} \leq \begin{cases}n|r| \frac{Q_{2 n-1}+6}{2}, & n \text { odd }, \\ n|r| \frac{Q_{2 n-1}+2}{2}, & n \text { even }\end{cases}
$$

where $\|\cdot\|_{2}$ is the spectral norm and $Q_{n}$ denotes the $n$th Pell-Lucas number.

Proof Since $A=C_{r}\left(Q_{0}^{2}, Q_{1}^{2}, \ldots, Q_{n-1}^{2}\right)$ is a $r$-circulant matrix, if the matrices $B=C_{r}\left(Q_{0}, Q_{1}\right.$, $\left.\ldots, Q_{n-1}\right)$ and $C=C\left(Q_{0}^{2}, Q_{1}^{2}, \ldots, Q_{n-1}^{2}\right)$ we get $A=B \circ C$; thus, we obtain

$$
\|A\|_{2} \leq \begin{cases}n|r| \frac{Q_{2 n-1}+6}{2}, & n \text { odd } \\ n|r| \frac{Q_{2 n-1}+2}{2}, & n \text { even }\end{cases}
$$

\section{Competing interests}

The authors declare that they have no competing interests.

\section{Authors' contributions}

All authors contributed equally to the writing of this paper. All authors read and approved the final manuscript.

\section{Author details}

'Science Faculty, Selcuk University, Konya, 42031, Turkey. ${ }^{2}$ Semsi Tebrizi Anatolian Religious Vocational High School, Konya, Turkey.

\section{Acknowledgements}

The authors wish to express their heartfelt thanks to the referees for their detailed and helpful suggestions for revising the manuscript.

Received: 29 September 2015 Accepted: 1 February 2016 Published online: 16 February 2016

\section{References}

1. Bahşi, M, Solak, S: On the norms of $r$-circulant matrices with the hyper-Fibonacci and Lucas numbers. J. Math. Inequal. 8(4), 693-705 (2014)

2. Bozkurt, D, Tam, TY: Determinants and inverses of $r$-circulant matrices associated with a number sequence. Linear Multilinear Algebra (2014). doi:10.1080/03081087.2014.941291

3. Shen, $\mathrm{S}, \mathrm{Cen}, \mathrm{J}$ : On the bounds for the norms of $r$-circulant matrices with the Fibonacci and Lucas numbers. Appl. Math. Comput. 216, 2891-2897 (2010)

4. Shen, S, Cen, J: On the spectral norms of $r$-circulant matrices with the $k$-Fibonacci and $k$-Lucas numbers. Int. J. Contemp. Math. Sci. 5(12), 569-578 (2010)

5. He, C, Ma, J, Zhang, K, Wang, Z: The upper bound estimation on the spectral norm $r$-circulant matrices with the Fibonacci and Lucas numbers. J. Inequal. Appl. (2015). doi:10.1186/s13660-015-0596-5

6. Halici, S: On some inequalities and Hankel matrices involving Pell, Pell-Lucas numbers. Math. Rep. 65(15), 1-10 (2013)

7. Koshy, T: Pell and Pell-Lucas Numbers with Applications. Springer, Berlin (2014)

8. Horn, RA, Johnson, CR: Topics in Matrix Analysis, pp. 333-335. Cambridge University Press, Cambridge (1991)

\section{Submit your manuscript to a SpringerOpen ${ }^{\circ}$ journal and benefit from:}

- Convenient online submission

Rigorous peer review

- Immediate publication on acceptance

- Open access: articles freely available online

- High visibility within the field

- Retaining the copyright to your article 